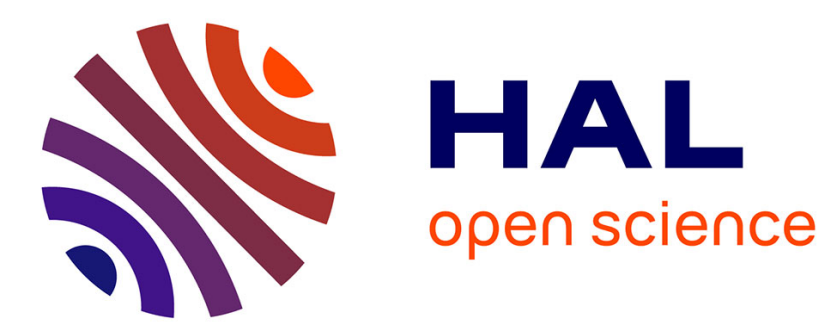

\title{
Logic gates operated by bipolar photoelectrochemical water splitting
}

Gabriel Loget, Gaozeng Li, Bruno Fabre

\section{To cite this version:}

Gabriel Loget, Gaozeng Li, Bruno Fabre. Logic gates operated by bipolar photoelectrochemical water splitting. Chemical Communications, 2015, 51 (55), pp.11115-11118. 10.1039/C5CC03811A . hal01166773

\section{HAL Id: hal-01166773 \\ https://hal-univ-rennes1.archives-ouvertes.fr/hal-01166773}

Submitted on 20 Oct 2015

HAL is a multi-disciplinary open access archive for the deposit and dissemination of scientific research documents, whether they are published or not. The documents may come from teaching and research institutions in France or abroad, or from public or private research centers.
L'archive ouverte pluridisciplinaire HAL, est destinée au dépôt et à la diffusion de documents scientifiques de niveau recherche, publiés ou non, émanant des établissements d'enseignement et de recherche français ou étrangers, des laboratoires publics ou privés. 


\title{
Logic Gates Operated by Bipolar Photoelectrochemical Water Splitting
}

\author{
G. Loget,* Gaozeng Li and Bruno Fabre*
}

Institut des Sciences Chimiques de Rennes, UMR 6226 (MaCSE) CNRS

Université de Rennes 1, Campus de Beaulieu, 35042 Rennes Cedex, France

* corresponding authors : gabriel.loget@univ-rennes1.fr ; fabre@univ-rennes1.fr

\begin{abstract}
A new approach for the design of logic gates that do not involve chemical inputs is presented here.

This concept is based on the polarization of a light-sensitive interface. AND and OR logic gates, working with cheap reactants, which locally triggered water splitting half reactions, were designed and operated.
\end{abstract}

The transfer of the fundamental concepts of microelectronics and integrated circuits to chemical and miniaturized fluidic systems is attracting a tremendous interest.1-7 The design, fabrication and operation of components allowing the processing and computation of signals in these media are therefore becoming very active areas of research. There has been a growing interest on the fabrication of molecular,2-4,8 microfluidic,5,6,9-11 and electrochemical devices12-17 able to control signals or perform binary operations. In typical molecular logic gates, the operations are performed by molecules or proteins either in solution8,18-21 or immobilized on a surface4,22 that generate a detectable chemical output only in a presence of certain external input signals (e.g. optical or chemical). Besides, microfluidic-based logic operations are often based on fluids or bubbles inputs, generating optical outputs5 or specific patterns.10 Few examples of electrochemical logic gates have been reported, most of them involving redox-active molecules immobilized on an electrode and generating optical or electrical outputs.12-17 Other appealing examples of electrochemical Boolean operators were reported by Berggren et al. who used the conductivity change of a conducting 
polymer,23 Amatore et al. who designed "artificial neurons" based on paired-band microelectrode assemblies 24 and Crooks et al. who conceived a microfluidic device generating electrochemiluminescence (ECL).25

In this context, we wish to report herein a novel approach towards logic gates operated by bipolar electrochemistry. Bipolar electrochemistry is a phenomenon that allows the generation of electrochemical reactions on the surface of conductive objects without the use of electrical wires.26,27 It is currently attracting considerable attention in the areas of analytical chemistry,28-32 materials science,33-41 motion generation $42-45$ and seawater desalination. 46 This phenomenon is particularly well suited for the implementation of miniaturized electrochemical systems in fluidic devices because of its inherent "wireless" nature.26,27 So far, the unique example of logic operators based on the use of bipolar electrochemistry was reported by the group of Crooks. 47 Their devices were operated by electrical inputs and involved the use of ruthenium-based luminescent complexes and an amine co-reactant for the generation of ECL as an output.48 The bipolar electrochemical splitting of water was reported by Fleischmann et al.49 and applied recently for steering conducting objects 43,44 and for the synthesis of Janus particles. 50 So far, only little interest 51,52 has been given to the use of semiconductor (SC) bipolar electrodes (BEs). It is worth noting that Ongaro et al. recently reported the electric field-assisted photoreduction of metal on TiO2 nanofibers.53
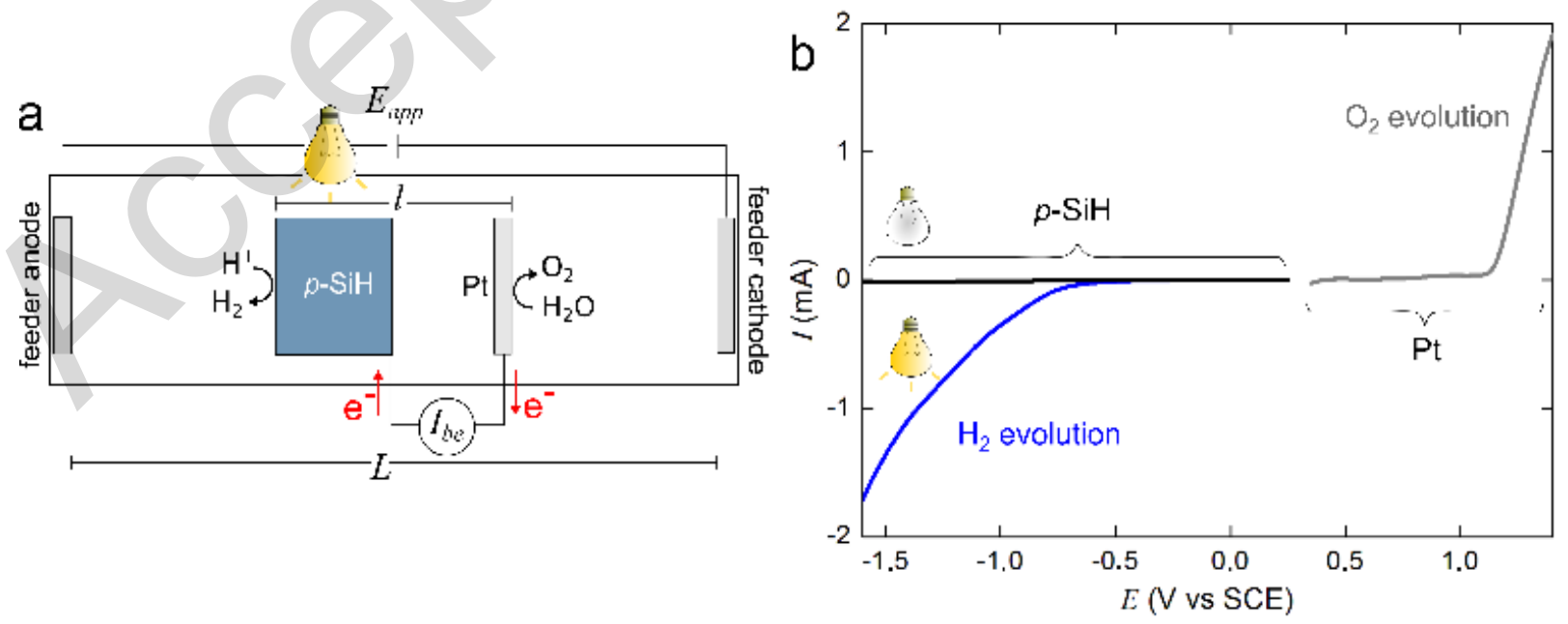
Fig 1. a) Scheme depicting the cell used for photoelectrochemical experiments performed with a p$\mathrm{SiH} / \mathrm{Pt} \mathrm{BE} . \mathrm{b}$ ) Voltammetric curves showing the anodic behavior of Pt (grey) and the cathodic behavior of $\mathrm{p}$-Si with (blue) and without (black) illumination. The curves were obtained at $50 \mathrm{mV} . \mathrm{s}-1$ in $50 \mathrm{mM} \mathrm{H} 2 \mathrm{SO} 4$.

We introduce here a new concept for the design of bipolar photoelectrochemical logic gates using a $\mathrm{SC}$ surface as a reactive pole, which allows for the first time to use light as an input signal. The fabrication and operation of AND and OR logic gates are described, that operate with the bipolar photoelectrochemical splitting of water and do not require direct connections. These devices can be very easily implemented and function only with water and a low concentration of supporting electrolyte (in the $\mathrm{mM}$ range).

In a typical bipolar electrochemistry experiment, a conducting object is placed in an electrolytic solution between two feeder electrodes, as shown in Fig. 1a. This object can be a conductive surface (e.g. a metal wire) or junctions. The application of a potential Eapp between the two feeder electrodes generates a potential drop in the solution and therefore leads to the generation of a polarization potential ?V along the object surface, which is maximal between its two ends.26,27 The value of this maximum polarization potential, ? $\mathrm{Vmax}$, is given by the following relation:

$$
\llbracket \Delta V \rrbracket \_m a x=E \_a p p / L \times 1 \times \theta
$$

with $L$ being the distance between the feeder electrodes $(4.6 \mathrm{~cm})$, I the characteristic length of the conducting surface and a dimensionless constant that depends on the potential losses of the cell.34 If ? $V \max$ is sufficiently high, electrochemical reactions can be toposelectively triggered: oxidation at the anodic pole (the pole facing the feeder cathode) and reduction at the cathodic pole (the pole facing the feeder anode). The conducting surface is then behaving at the same time as an anode and a cathode, that is, a BE.26,27 An important aspect is that electron production and consumption must be equal at both sides of the BE in order to maintain charge neutrality. In the present work, the logic 
gates were designed on the basis of the configuration shown in Fig. 1a, with a split-BE26 composed of an oxide-free, hydrogen-terminated p-type silicon ( $\mathrm{p}-\mathrm{SiH})$ cathodic pole and a Pt anodic pole, electrically connected outside to the cell. This configuration allowed to easily measure the current Ibe flowing through the BE with an ammeter. All the reported experiments were performed in 50 $\mathrm{mM}$ aq $\mathrm{H} 2 \mathrm{SO} 4$ in the dark and under irradiation with a halogen lamp.

First, both materials used for the split-BE were investigated separately by voltammetry in a classical three-electrode electrochemical cell. The anodic response of Pt was independent of light and, as shown in Fig. 1b, displayed an anodic current starting at $1.1 \mathrm{~V}$ vs SCE, which corresponds to $\mathrm{O} 2$ evolution:

$2 \mathrm{H} 2 \mathrm{O} \rightarrow \mathrm{O} 2+4 \mathrm{H}++4 \mathrm{e}-$

Unlike metals, the electrical properties of SC electrodes can be dramatically changed by light. For instance, p-type Si requires the generation of minority carriers (i.e. electrons) to operate as a photocathode, therefore irradiation with a wavelength higher than the material band gap $(1.1 \mathrm{eV})$ is necessary in order to promote electrochemical reduction reactions at its surface.54 Consistent with that, no cathodic current was generated in the dark at $\mathrm{p}-\mathrm{SiH}$ (black curve in Fig. 1b) while a significant photocurrent beginning at -0.6 V vs SCE was observed under illumination (blue curve), which corresponds to proton reduction:

$4 \mathrm{H}++4 \mathrm{e}-\rightarrow 2 \mathrm{H} 2$ 
These preliminary data suggest that reduction of water cannot occur at the cathodic pole $(\mathrm{p}-\mathrm{SiH})$ of the $B E$ in the dark. Because the charge consumption must be equal at both sides of the $B E, 26,27$ this blocking behavior should also prevent oxidation reaction to occur at the anodic pole and therefore no current should flow through the object. On the contrary, bipolar reactions should be possible under illumination, if the applied electric field is sufficiently high to generate a ?Vmax $>1.7 \mathrm{~V}$ between the ends of the $\mathrm{BE}$, corresponding to the thermodynamic value that must be overcame in order to trigger reactions (2) and (3), as determined by Fig. $1 \mathrm{~b}$. These conditions should lead to the generation of a faradaic current Ibe flowing through the BE, as illustrated in Fig. 1a.
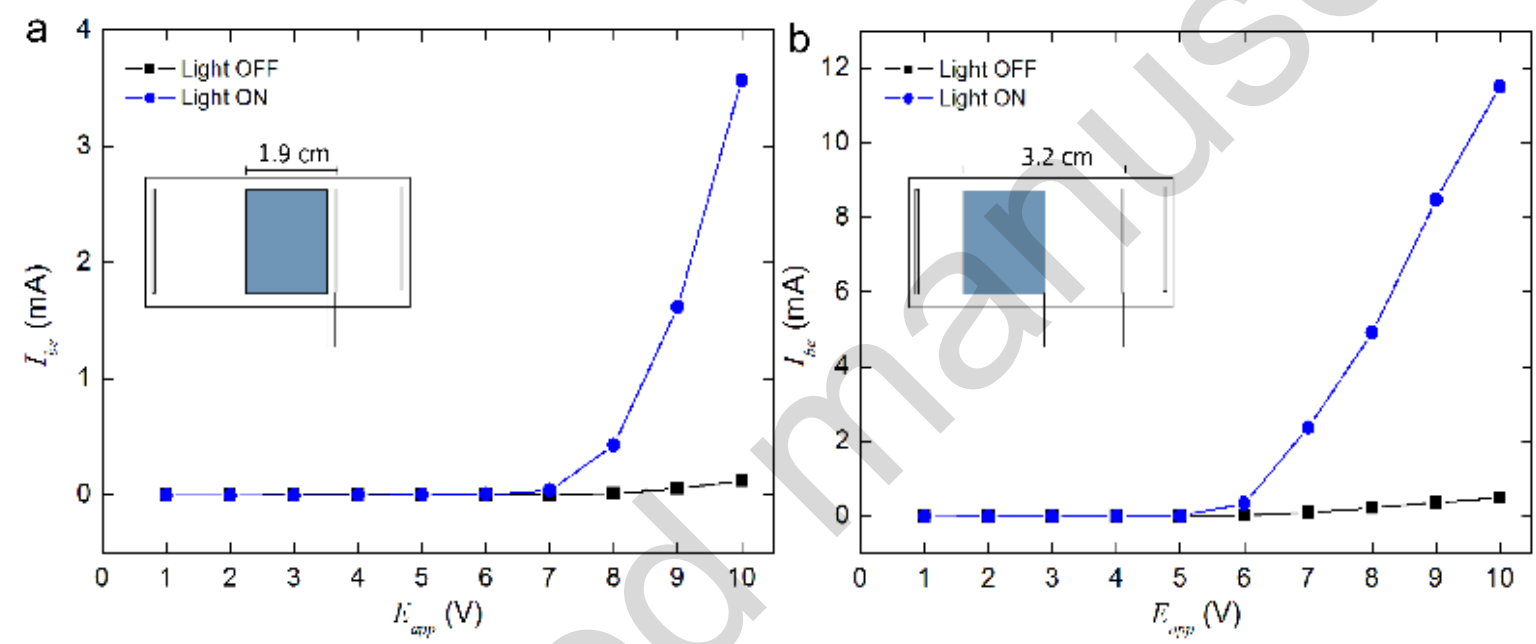

Fig 2. Curves showing the current flowing through the $B E$, Ibe as a function of the potential applied between the feeder electrodes Eapp in the dark (black curves) and under illumination (blue curves) for a) $1.9 \mathrm{~cm}$-long and b) $3.2 \mathrm{~cm}$-long BE. The insets are schemes representing the corresponding cells.

In order to assess thermodynamic and kinetic parameters directly on the $\mathrm{BE}$, Ibe measurements were performed while imposing different values of Eapp, with and without illumination. Two characteristic BE lengths, $I 1=1.9 \mathrm{~cm}$ and $\mathrm{I} 2=3.2 \mathrm{~cm}$, were used in this work. From the data shown in Fig. 2 , it is obvious that a similar trend was obtained for both BEs. Under illumination, lbe was equal to zero until values Eapp1 $=7 \mathrm{~V}$ and Eapp2 $=5 \mathrm{~V}$ were respectively reached for $\mathrm{I} 1$ and $\mathrm{I} 2$ and then $\mathrm{Ibe}$ increased with Eapp. These threshold values correspond to the potentials to apply in order to 
generate a ? $\mathrm{Vmax}$ of $1.7 \mathrm{~V}$ and locally trigger reactions (2) and (3). The highest value, Eapp1 was found for the smallest I value, which is well in line with eq. (1). This relation allowed us to calculate that $\sim 50 \%$ of the applied electric field is effectively restituted to the BE (average $1=0.54$ ), due to losses that may be attributed to field perturbation in the cell or potential drops at the solid/liquid or solid/solid interfaces. As expected, dark currents were found in both cases much lower than photocurrents ( 25 times for Eapp $=10 \mathrm{~V}$, see Fig S1) thanks to the insulating behavior of $p$-SiH under these conditions. These data demonstrate that illumination and applied potential generate large current modifications in these BEs that can be easily measured. Therefore, such a configuration is very well-suited for the fabrication of two-input logic gates, which will be now described.

The two inputs can be defined as Eapp $(\ln 1)$ and illumination $(\ln 2)$, whereas the current Ibe can be considered as the output signal. Based on data of Fig. $2 \mathrm{a}$, we decided to set $\ln 1=0$ for Eapp $=7 \mathrm{~V}$ and $\ln 1=1$ for Eapp $=10 \mathrm{~V}$, in order to obtain a sufficiently high output signal when all the inputs will be equal to 1 . The threshold output value was arbitrary set to $1.5 \mathrm{~mA}$. The transient signals $\ln 1$ and $\ln 2$ and the resulting outputs for both systems are shown in Fig. 3a-d and summarized in the corresponding truth tables (Fig. 3f,g). The $1.9 \mathrm{~cm}$-long BE (Fig. 3c) exhibited an output signal "1" (Ibe $>1.5 \mathrm{~mA}$ ) only when $\ln 1=\ln 2=1$. All the other combinations yield an output signal of "0" (Ibe $<1.5$ $\mathrm{mA})$. Therefore, this system acted as an AND gate. As shown in Fig. 3e, generation of $\mathrm{H} 2$ and $\mathrm{O} 2$ bubbles was observed at both reactive poles of the BE when Output = "1", confirming the triggering of reactions ( 2 ) and ( 3 ). The release of $\mathrm{H} 2$ bubbles from the $\mathrm{BE}$ surface caused the current fluctuations that are visible in the output signal (Fig. 3c). In contrast, the $3.2 \mathrm{~cm}$-long BE (Fig. 3d) was found to be always in its state "1" when illuminated, which is in good agreement with the data shown in Fig. 2b. Even if the latter configuration is not so beneficial in terms of Boolean logic (a YES gate, performing the operation Output $=\ln 2$ ), it will be very useful in the following for the design of an OR logic gate. 


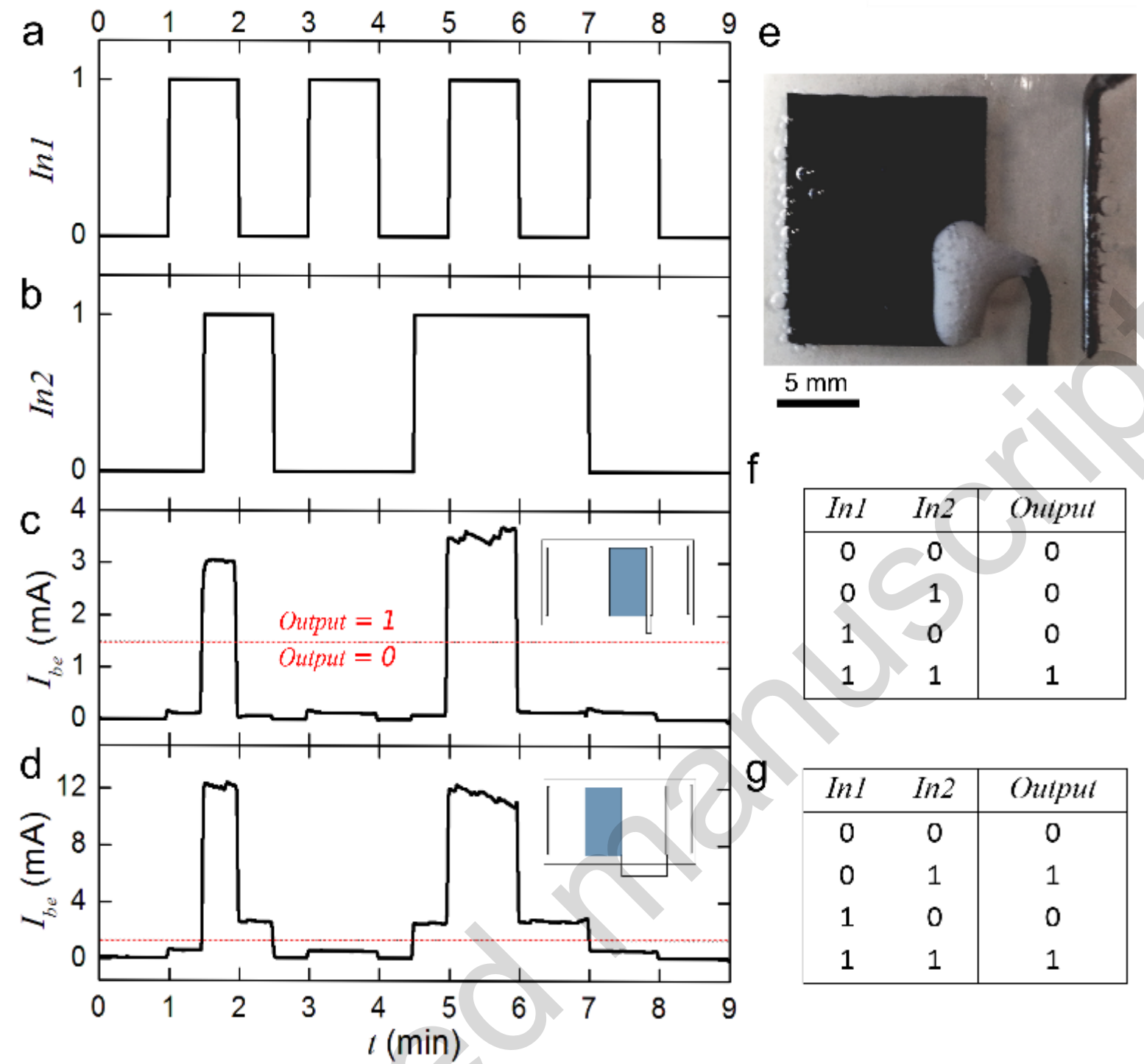

Fig. 3. Time-dependent profiles of the applied input signals a) $\ln 1(\ln 1=0$ for Eapp $=7 \mathrm{~V}$ and $\ln 1=1$ for Eapp $=10 \mathrm{~V}), \mathrm{b}) \ln 2(\ln 2=0$ when the light is OFF and $\ln 2=1$ when the light is ON) and the output Ibe measured for c) a $1.9 \mathrm{~cm}$-long BE acting as an AND gate and d) a $3.2 \mathrm{~cm}$-long BE. Both BEs are composed of a p-SiH cathodic pole and a Pt anodic pole. The red line indicates the threshold current of $1.5 \mathrm{~mA}$. e) Photograph of the split BE showing the evolution of $\mathrm{H} 2$ and $\mathrm{O} 2$ bubbles at the cathodic pole (left) and at the anodic pole (right), respectively. f,g) Truth tables summarizing the results obtained in f) Fig. 3c and g) Fig. 3d. 
For comparison, split BEs only composed of Pt wires were also investigated and were found to perform the following operations: Output = "1" whatever the states of In1 and In2 for I = $3.2 \mathrm{~cm}$ (see Fig. S2 in the Supporting Information) and Output $=\ln 1 \mathrm{for} I=1.9 \mathrm{~cm}$, as shown in Fig. 4c. These results were expected because the conductivity of metals like Pt is not sensitive to light, therefore the output was always independent of $\ln 2$. The latter configuration was particularly attractive since it allowed to reach the combination that was missing in the truth table of Fig. $3 g$ in order to get an OR gate, (i.e. Output $=1$ when $\ln 1=1$ and $\ln 2=0$ ). An interesting strategy to combine the $\mathrm{BE}$ configurations of Fig. $3 d$ and $4 c$ was to use a hybrid BE made of a light-sensitive component as well as a component non-sensitive to light. The simplest design, depicted in Fig. 4e,f, consisted in the connection in parallel of a p-SiH surface with a Pt wire. The output signal of such a BE with the corresponding truth table are shown in Fig. 4d,h. As expected, this BE acted as an OR gate with the Pt wire being the cathodic pole when $\ln 1=1$ and $\ln 2=0$, and $p$-SiH being the cathodic pole when $\ln 1=$ 0 and $\ln 2=1$. These experiments demonstrate that AND and OR logic gates operated by bipolar photoelectrochemical water splitting can be designed and easily operated. 


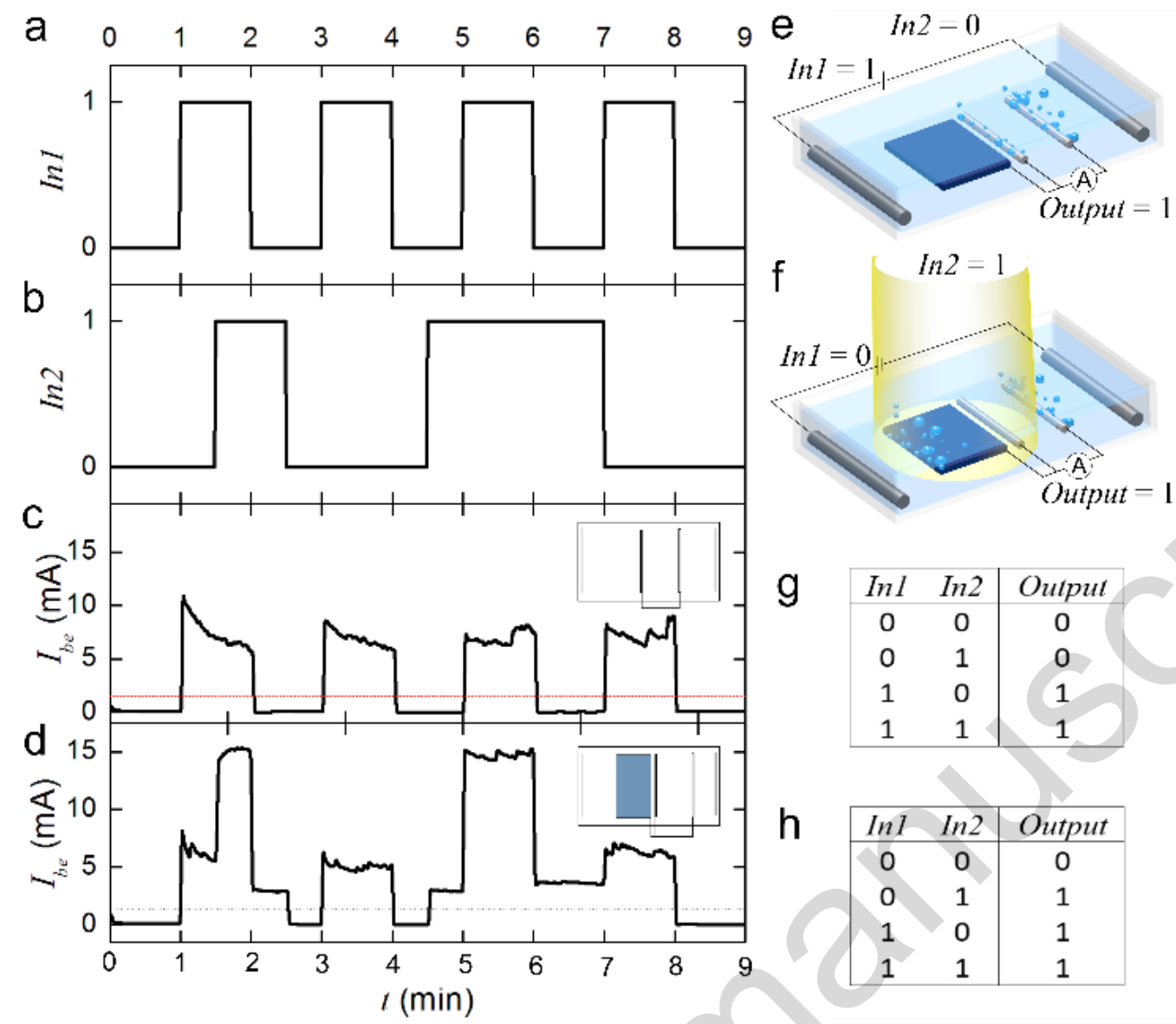

Fig. 4. Time-dependent profiles of the applied input signals a) $\ln 1(\ln 1=0$ for Eapp $=7 \mathrm{~V}$ and $\ln 1=1$ for Eapp $=10 \mathrm{~V}), \mathrm{b}) \ln 2(\ln 2=0$ when the light is OFF and $\ln 2=1$ when the light is ON $)$ and the output Ibe measured for c) a $1.9 \mathrm{~cm}$-long BE with two Pt reactive poles and d) a $3.2 \mathrm{~cm}$-long BE com-posed of a $\mathrm{p}-\mathrm{SiH} / \mathrm{Pt}$ cathodic pole and a $\mathrm{Pt}$ anodic pole acting as an OR gate. The red line indicates the threshold current of $1.5 \mathrm{~mA}$. e,f) Cell schemes showing the OR gate operating when e) $\ln 1=1$ and $\ln 2$ $=0$ and f) $\ln 1=0$ and $\ln 2=1 . \mathrm{g}, \mathrm{h}$ ) Truth tables summarizing the results obtained in g) Fig. $4 \mathrm{c}$ and $\mathrm{h}$ ) Fig. 4d.

The percentages of current flowing through the BEs were calculated for the AND and the OR logic gates and are reported in Table S1. Interestingly, they varied from values smaller than $0.4 \%$ when Output = "0" to values greater than $5 \%$ when Output = "1", with a maximum of $22 \%$ for the OR gate when $\ln 1=\ln 2=1$. In order to assess the time stability of the involved surfaces, cycling tests were performed with the AND gate by imposing switching cycles to $\ln 1$ while keeping $\ln 2=1$. As shown in 
Fig. 5, Ibe was found to be constant over numerous switching cycles. Such a stability of the p-SiHbased BE is thus very promising for future integration of the logic gates in fluidic devices.

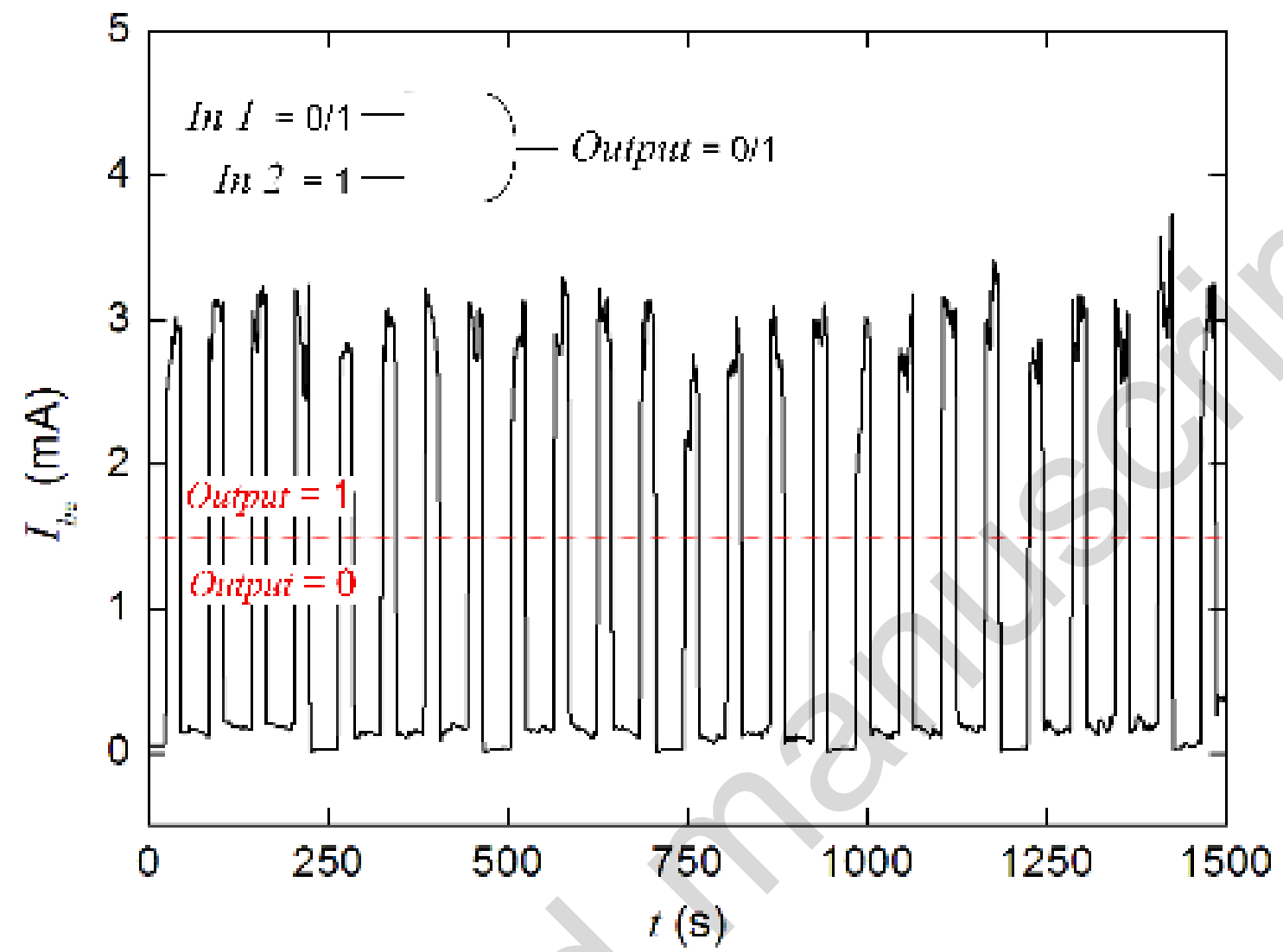

Fig. 5. Curve showing the time evolution of the output signal Ibe for the AND gate when $\ln 1$ is switched ON and OFF. The red line indicates the threshold current $1.5 \mathrm{~mA}$.

\section{Conclusions}

In this work, we have demonstrated a new strategy for the design of electrochemical logic gates working with optical and electrical inputs. Robust and stable AND and OR gates, operated with cheap reactants (i.e. acidic water) were fabricated. The inputs were mediated by electric and electromagnetic fields and the gates did not required direct connection. Thanks to their "wireless" implementation, these devices should be easily downscaled and therefore could be operated with LEDs in miniaturized devices such as micro-fluidic channels and lab-on-chips with suitable electric 
fields (see eq. 1). It should be also possible to combine several gates in a single fluidic device and use the current flowing through the BE to perform additional logic operations or to supply integrated electronic devices.44 Additionally, a signal other than the bipolar current (i.e. bubble production or the generation of an optically-active compound at a reactive pole) could be considered as output, in order to minimize the electrical connections. In this first report, $\mathrm{SC} /$ metal BEs were employed but it is evident that our approach can be extended to $\mathrm{p}-\mathrm{SC} / \mathrm{n}$-SC BEs which will enlarge the panel of available operations and reduce the cost of the devices. Considering that a very large panel of materials and co-catalysts combinations can be used as BEs and that the electrolyte composition will have a direct effect on the output values, we believe that this work pave the way to a broad family of signal processing devices for chemical and fluidic systems.

\section{Notes and references}

1 Y. Amir, E. Ben-Ishay, D. Levner, S. Ittah, A. Abu-Horowitz and I. Bachelet, Nat. Nanotechnol., $2014,9,353-357$.

2 K. Szaciłowski, Chem. Rev., 2008, 108, 77-84.

3 P. de Silva and S. Uchiyama, Nat. Nanotechnol., 2007, 2, 399-410.

4 G. De Ruiter and M. E. Van Der Boom, Acc. Chem. Res., 2011, 44, 563-573.

Nam, J. Yoon and S. Park, Angew. Chem. Int. Ed., 2008, 47, 872-876.

6

B. Mosadegh, C.-H. Kuo, Y.-C. Tung, Y. Torisawa, T. Bersano-Begey, H. Tavana and S.

Takayama, Nat. Phys., 2010, 6, 433-437.

7

F. Meng, Y.-M. Hervault, Q. Shao, B. Hu, L. Norel, S. Rigaut and X. Chen, Nat.

Commun., 2014, 5, 3023. 
2015, 2015,11,1811-1817.

9

T. Thorsen, S. J. Maerkl and S. R. Quake, Science, 2002, 298, 580-584.

10

M. Prakash and N. Gershenfeld, Science, 2007, 315, 832-835.

Mahadevan and G. M. Whitesides, J. Am. Chem. Soc., 2009, 131, 12420-12429.

12

B. Fabre, Y. Li, L. Scheres, S. P. Pujari and H. Zuilhof, Angew. Chem. Int. Ed., 2013, 52, $12024-12027$.

3918-3920. Int. Ed., 2006, 45, 3143-3146.

Chem. Soc., 2011, 133, 11641-11648. $2012,51,12477-12481$. 
110, 8548-8553.

22

G. De Ruiter, E. Tartakovsky, N. Oded and M. E. Van Der Boom, Angew. Chem. Int.

Ed., 2010, 49, 169-172.

D. Nilsson, N. Robinson, M. Berggren and R. Forchheimer, Adv. Mater., 2005, 17, 353-358.

Chem., 2014, 86, 3138-3145. 2358. 2523. 
Chem. Sci., 2014, 5, 1961-1966.

36

G. Loget, S. So, R. Hahn and P. Schmuki, J. Mater. Chem. A, 2014, 2, 17740-17745.

37

G. Loget and P. Schmuki, Langmuir, 2014, 30, 15356-15363.

38

J.-C. Bradley, H.-M. Chen, C. J., J. Eckert, K. Ernazarova, T. Kurzeja, M. Lin, M. McGee,

W. Nadler and S. G. Stephens, Science, 1997, 389, 268-271.

39

S. Ramakrishnan and C. Shannon, Langmuir, 2010, 26, 4602-4606.

40

N. Shida, Y. Koizumi, H. Nishiyama, I. Tomita and S. Inagi, Angew. Chem., 2015, 127,

3994-3998.

41

M. Wood and B. Zhang, ACS Nano, 2015, 9, 2454-2464.

42

G. Loget and A. Kuhn, J. Am. Chem. Soc., 2010, 132, 15918-15919.

43

G. Loget and A. Kuhn, Nat. Commun., 2011, 2, 535.

44

J. Roche, S. Carrara, J. Sanchez, J. Lannelongue, G. Loget, L. Bouffier, P. Fischer and A.

Kuhn, Sci. Rep., 2014, 4, 6705.

45

A. Srinivasan, J. Roche, V. Ravaine and A. Kuhn, Soft Matter, 2015,

DOI:10.1039/C5SM00273G.

46

K. N. Knust, D. Hlushkou, R. K. Anand, U. Tallarek and R. M. Crooks, Angew. Chem. Int.

Ed., 2013, 52, 8107-8110.

47

B. Y. Chang, J. a. Crooks, K. F. Chow, F. Mavré and R. M. Crooks, J. Am. Chem. Soc.,

2010, 132, 15404-15409.

48

W. Miao, Chem. Rev., 2008, 108, 2506-2553. 
$6392-6400$.

50

G. Loget, J. Roche, E. Gianessi, L. Bouffier and A. Kuhn, J. Am. Chem. Soc., 2012, 134,

$20033-20036$.

51

S. Cattarin and M. M. Musiani, J. Electrochem. Soc., 1995, 142, 3786.

52

E. Said, N. D. Robinson, D. Nilsson, P.-O. Svensson and M. Berggren, Electrochem.

Solid-State Lett., 2005, 8, H12-H16.

53 M. Ongaro, J. Roche, A. Kuhn and P. Ugo, ChemElectroChem, 2014, 1, 2048-2051.

54 X. G. Zhang in Electrochemistry of Silicon and its Oxide, Kluwer Academic/Plenum Publishers, New York, 2001. 